\title{
All-Dielectric Meta-optics for High-Efficiency Independent Amplitude and Phase Manipulation
}

\section{Brian Raeker}

University of Michigan at Ann Arbor https://orcid.org/0000-0002-5359-6878

\section{Hanyu Zheng}

Vanderbilt University

\section{You Zhou}

Vanderbilt University https://orcid.org/0000-0002-5810-2347

\section{Ivan Kravchenko}

Oak Ridge National Laboratory https://orcid.org/0000-0003-4999-5822

\section{Jason Valentine}

Vanderbilt University https://orcid.org/0000-0001-9943-7170

\section{Anthony Grbic ( $\nabla$ agrbic@umich.edu )}

University of Michigan at Ann Arbor https://orcid.org/0000-0002-3461-1083

\section{Article}

Keywords: Metasurfaces, Transmitted Amplitude, Combined Beam-forming, Splitting Devices, Threedimensional Holograms

Posted Date: February 26th, 2021

DOI: https://doi.org/10.21203/rs.3.rs-253126/v1

License: (1) (i) This work is licensed under a Creative Commons Attribution 4.0 International License. Read Full License

Version of Record: A version of this preprint was published at Advanced Photonics Research on December 26th, 2021. See the published version at https://doi.org/10.1002/adpr.202100285. 


\section{All-Dielectric Meta-optics for High-Efficiency Independent Amplitude and Phase Manipulation}

\section{Author Information}

\section{Affiliations}

Department of Electrical Engineering and Computer Science, University of Michigan, Ann Arbor, MI, 48109 USA

Brian O. Raeker*, Anthony Grbic ${ }^{*}$

Department of Electrical Engineering and Computer Science, Vanderbilt University, Nashville, TN, 37212 USA

Hanyu Zheng*

Photonics Initiative, Advanced Science Research Center, City University of New York, New York, NY, 10031, USA

You Zhou

Center for Nanophase Materials Sciences, Oak Ridge National Laboratory, Oak Ridge, Tennessee 37831, USA

Ivan I. Kravchenko

Department of Mechanical Engineering, Vanderbilt University, Nashville, TN, 37212 USA

Jason Valentine ${ }^{\dagger}$

\section{Author Contributions}

*these authors contributed equally to this work.

B.O.R. developed device designs, conducted simulations, and wrote the manuscript. H.Z. and Y.Z.

performed device fabrication and experimental measurements. I.I.K. performed the material growth. J.V.

and A.G. conceived the research concepts and supervised the project. All authors discussed research results and participated in preparing the manuscript.

Corresponding author

Correspondence to: ${ }^{\star}$ Anthony Grbic, agrbic@umich.edu

†Jason Valentine, jason.g.valentine@vanderbilt.edu 


\section{Abstract}

Metasurfaces, composed of subwavelength scattering elements, have demonstrated remarkable control over the transmitted amplitude, phase, and polarization of light. However, manipulating the amplitude upon transmission has required loss if a single metasurface is used. Here, we describe highefficiency independent manipulation of the amplitude and phase of a beam using two lossless phase-only metasurfaces separated by a distance. With this configuration, we experimentally demonstrate optical components such as combined beam-forming and splitting devices, as well as those for forming complexvalued, three-dimensional holograms. The compound meta-optic platform provides a promising approach for achieving high performance optical holographic displays and compact optical components, while exhibiting a high overall efficiency.

\section{Introduction}

Controlling the amplitude, phase, and polarization of an optical field is essential to many applications in a broad range of scientific and industrial areas. Conventionally, this is achieved using a sequence of optical components such as lenses, polarizers, gratings, and amplitude masks. Such an approach results in physically large systems as well as reduced efficiency, especially if transmission loss is used to control the amplitude profile of the optical wave. As technological demand increases for compact and high-efficiency optical systems, optical metasurfaces offer a path toward satisfying such requirements. Metasurfaces are two-dimensional arrays of subwavelength scatterers, designed to exhibit remarkable control over the transmitted phase, amplitude, and polarization of light. Specifically, the ability to locally control the transmission characteristics provides a powerful approach to realizing flat optical components and systems with improved performance ${ }^{1-4}$. This has been shown through a variety of devices, including flat lenses ${ }^{5,6}$, beam-splitters ${ }^{7-12}$, holograms ${ }^{13-17}$, augmented reality displays ${ }^{18}$, highdefinition displays ${ }^{19}$, image differentiation ${ }^{20}$, and compact optical spectrometers ${ }^{21}$. However, optical applications requiring spatial manipulation of the amplitude profile have relied on reflection ${ }^{22}$, absorption $^{23}$, or polarization conversion loss ${ }^{11,12,14-17}$, resulting in low efficiencies. 
Independently controlling the spatial amplitude and phase distributions of an electromagnetic wave is essential to forming optical components such as combined beam-former and splitter devices. Metasurface beam-splitters have been demonstrated through a variety of methods ${ }^{7-12}$, however the beam shape is not altered, and efficiency is reduced due to diffraction or the use of loss. Another use of amplitude and phase control is in forming three-dimensional holograms, which can be produced with high quality if a specific complex-valued field profile is formed ${ }^{16}$. Polarization loss has been used to form three-dimensional holograms ${ }^{14-17}$ by performing a spatially varying polarization conversion across the metasurface. Amplitude control is implemented by filtering the unconverted polarization component, while phase control is implemented via another metasurface parameter. While capable of producing highquality holographic images, the efficiency of such devices is low and application-specific due to reliance on polarization loss.

In this work, we experimentally demonstrate high-efficiency, independent amplitude and phase control over an optical field using compound meta-optics. Compound meta-optics are sequential metasurfaces separated by a distance and arranged along a common axis, which perform functions beyond the capabilities of individual metasurfaces ${ }^{24,25}$. The configuration of the compound meta-optic is shown in Figure 1(a). A process for designing paired phase-only metasurfaces was previously developed to implement independent amplitude and phase control ${ }^{24,25}$. This process was verified through full-wave simulation at near-infrared wavelengths to form high-efficiency combined beam-former and splitters and a high-quality, three-dimensional hologram ${ }^{25}$. Using this approach, the spatial separation of the metasurfaces enables high-efficiency amplitude control by redistributing the optical beam amplitude instead of using loss to form the desired amplitude distribution. Alternative methods have also been developed to optimize sequential metasurfaces for spatial complex-valued control over a wave. Optimizing metasurface unit cell parameters using the adjoint optimization method ${ }^{26}$, optimizing the plane wave spectrum ${ }^{27}$, and optimizing equivalent current distributions ${ }^{28}$, have been used to design sequential metasurface systems. Additionally, a variety of applications have been demonstrated with 
sequential metasurface devices: aberration correction ${ }^{29}$, optical retro-reflection ${ }^{30}$, full-color holography ${ }^{31}$, and optical diffractive neural networks ${ }^{32,33}$.
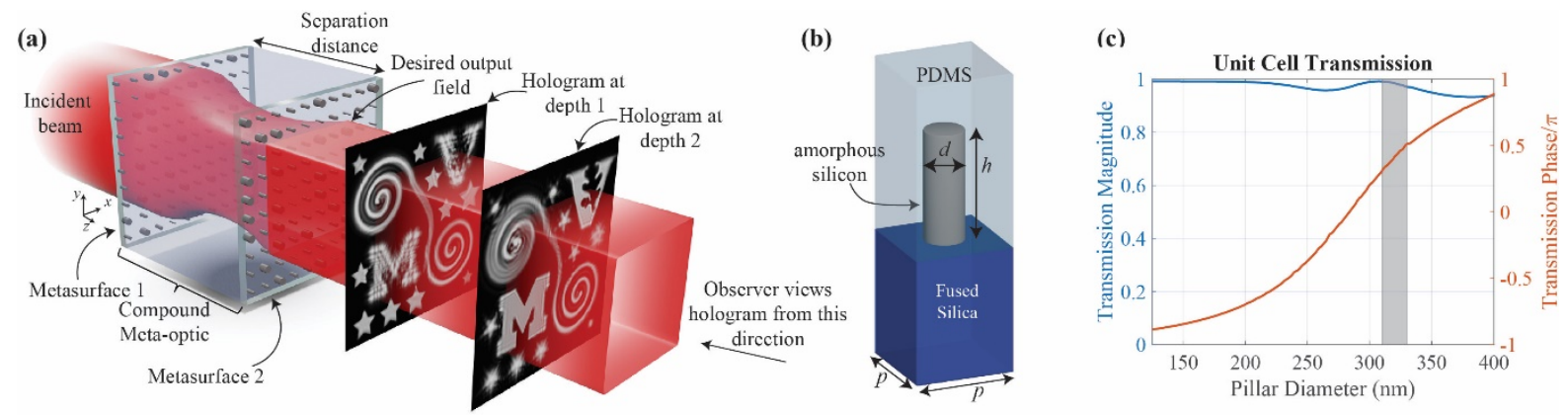

Figure 1: Diagram of the optical compound meta-optic implementing independent phase and amplitude control over an incident optical field. (a) Illustration of a compound meta-optic converting a uniform illumination into a three-dimensional, complex-valued hologram. Depth of the three-dimensional hologram is visualized when the output field is imaged at different depths by an observer facing the device. (b) A schematic of the metasurface unit cell. Each cell has a period $p=570 \mathrm{~nm}$, and contains an amorphous silicon nanopillar with a height $h=850 \mathrm{~nm}$. The nanopillar diameter is varied across the metasurface to implement the desired transmission phase profile. (c) The transmission characteristics of the unit cell under normally incident plane wave illumination and periodic boundary conditions. A phase coverage of $78 \%$ with transmission above 0.93 is achieved by varying the pillar diameter. Diameters of $310 \mathrm{~nm}-330 \mathrm{~nm}$ are not used in the three-dimensional hologram designs due to observed low transmission in fabrication, which impact the image quality.

The compound meta-optic is formed by two lossless phase-only metasurfaces separated by a physically short distance of homogeneous dielectric, as shown in Figure 1(a). Together, they provide the desired independent amplitude and phase control. Adding additional metasurfaces would allow more control over the optical field (e.g. multi-wavelength performance ${ }^{6,22,26}$, or diffractive neural networks ${ }^{26,32,33}$ for multi-input multi-output applications), but only two metasurfaces are necessary for amplitude and phase control at a single wavelength. As a proof of concept, we report experimental demonstrations of meta-optics that combine beam-forming and splitting, and produce high-quality, threedimensional holograms. The efficiency of the devices is high, only limited by the minor reflection from 
each metasurface. Here, we show the promise of optical compound meta-optics for high-efficiency holographic displays and optical components.

\section{Results}

\section{Concept and design of compound meta-optics.}

The proposed compound meta-optic devices consist of two phase-only metasurfaces, each impressing a phase discontinuity onto an incident wave. The design method has been developed and verified in simulation at microwave frequencies ${ }^{24}$ and near-infrared wavelengths ${ }^{25}$. The first metasurface applies a phase discontinuity onto the known incident wave. This phase shift is designed so that the desired electric field amplitude distribution is formed at a distance equal to the separation distance between the metasurfaces. However, the phase of this field profile is incorrect relative to the desired output phase profile. Therefore, the second metasurface applies a phase correction to form the desired complex-valued electric field distribution. Since the metasurfaces are assumed to be transparent, the amplitude distributions of the wave transmitted by metasurface 1 and incident on metasurface 2 are known from the incident and desired field profiles, respectively. The free parameters are the transmission phase distributions at each plane, which are optimized using a phase-retrieval algorithm that links the incident and output field amplitude distributions. Specifically, the Gerchberg-Saxton phase-retrieval algorithm ${ }^{34}$ is modified to operate over short distances instead of the near-to-far-field conversion typically used. The phase shift distribution of each metasurface is calculated as the difference between the phase distributions of the transmitted and incident fields.

$$
\begin{gathered}
\phi_{m s 1}=\phi_{t r 1}-\phi_{E_{i n c}} \\
\phi_{m s 2}=\phi_{E_{\text {out }}}-\phi_{\text {inc2 }}
\end{gathered}
$$


where $\phi_{E_{\text {inc }}}$ is the phase of the incident field, $\phi_{t r 1}$ is the phase profile of the transmitted field from metasurface 1, $\phi_{i n c 2}$ is the phase of the field incident on metasurface 2, and $\phi_{E_{\text {out }}}$ is the phase profile of the desired output field. The metasurfaces are then designed to implement $\phi_{m s 1}$ and $\phi_{m s 2}$ as a transmission phase shift of the form $e^{i \phi_{m s}}$ on the incident wave. Here, a time convention of $e^{-i \omega t}$ is assumed.

Each metasurface is a dense array of silicon nanopillars, which are commonly used to implement desired spatial phase and polarization modulations with high transmission efficiency ${ }^{1-4}$. Figure $1(\mathrm{~b})$ shows a schematic of the unit cell, with the silicon nanopillar embedded in a layer of polydimethylsiloxane (PDMS) at the interface of a fused silica handle wafer. Circular cross-sections of the nanopillars were

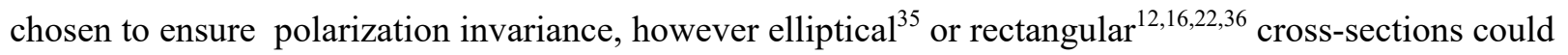
be used if polarization control is required. Variation of the pillar diameter $(d)$ provides control over the transmission phase. The silicon pillar height $(h=850 \mathrm{~nm})$, unit cell period $(p=570 \mathrm{~nm})$, and illuminating wavelength $\left(\lambda_{0}=1.3 \mu \mathrm{m}\right)$ have been chosen to provide high transmittance for a large transmission phase range. The locally periodic approximation ${ }^{37,38}$ is made to determine the unit cell transmission by assuming that the nanopillars are placed in a homogeneous array environment. This approximation is commonly used to simplify the design of unit cells in inhomogeneous arrays and is generally accurate if the unit cells are not strongly coupled (as is the case here). Figure 1(c) shows the transmission for the chosen dimensions as a function of pillar diameter. For these dimensions, a transmission phase coverage of $78 \%$ can be achieve with a transmission magnitude greater than 0.93 . Note that the pillar diameters of $125 \mathrm{~nm}-400 \mathrm{~nm}$ are used in the beam-splitter designs, but 310 $\mathrm{nm}-$ $330 \mathrm{~nm}$ are not used in the three-dimensional hologram design. This is due to observed low transmission for these diameters in measurement, which impacts image quality (additional details can be found in Supplementary Note 1). 
The phase shift profiles producing the desired amplitude and phase conversion of the input field are sampled at the unit cell periodicity and converted to distributions of nanopillars with the corresponding diameters. Each metasurface is then fabricated individually and aligned to form the compound meta-optic devices.

\section{Fabrication and characterization of compound meta-optics}

The meta-optic devices were fabricated using nanofabrication techniques developed to construct multi-metasurface devices ${ }^{6,22}$. First, an $850 \mathrm{~nm}$-thick layer of amorphous silicon was deposited onto a fused silica substrate. Each metasurface pattern was defined using electron beam lithography (EBL) and then nanopillars were formed using reactive ion etching (RIE) ${ }^{6,20,22}$. The metasurfaces were subsequently enclosed in a protective layer of PDMS. Figure 2(a) shows optical images of the meta-optic devices and one fabricated metasurface, and a scanning electron microscope image of the circular silicon nanopillars after RIE. Finally, the metasurfaces were spaced by a layer of PDMS and carefully aligned using translation stages to form the complete compound meta-optic, as shown in Figure 2(b). Accurate alignment between the two metasurfaces $(<1.5 \mu \mathrm{m}$ lateral misalignment $)$ is necessary to obtain the desired complex-valued field transformation. To achieve this, we developed hologram alignment marks formed by silicon arrays fabricated near each of the two metasurfaces (additional information can be found in Supplementary Note 2). After spatial alignment was achieved with the alignment marks, the alignment was further adjusted until the desired intensity image was formed at a distance beyond the meta-optic output. Misalignment introduces phase error to the output field distribution and the output field phase defines the propagation behavior of the wave, so the alignment improves as the observed intensity image improves. 
(a)

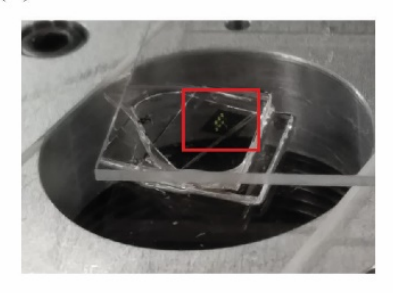

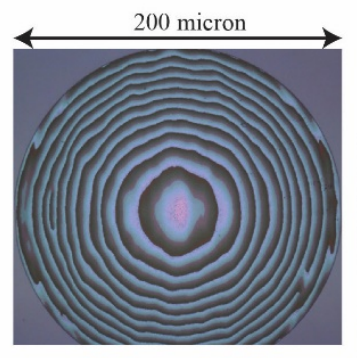

(b)

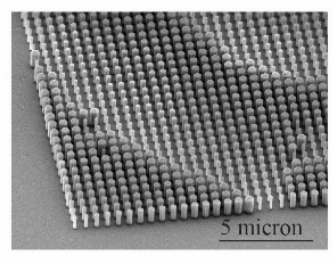

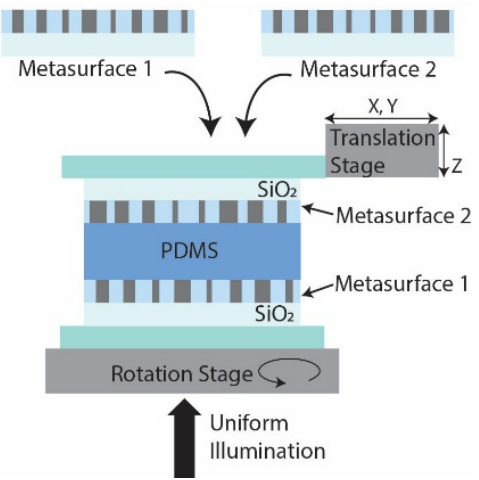

Figure 2: Images of a fabricated meta-optic and schematic of meta-optic assembly. (a) Left: An optical image of the fabricated meta-optic device, where the two metasurface layers are being aligned. Middle: Optical image of a fabricated metasurface, showing the variation in pillar diameter as a change in color. Right: A scanning electron microscope image of a portion of one metasurface, showing the array of silicon nanopillars. (b) Schematic of the meta-optic assembly, alignment, and characterization process. Each metasurface is fabricated individually and then aligned to form the meta-optic device.

The compound meta-optics were characterized using an unpolarized supercontinuum laser whose beam is passed through a monochromator to select the desired wavelength. The resulting beam overfilled the meta-optic footprint to form the desired uniform illumination. The meta-optic performs the required spatial amplitude and phase manipulation to form the desired complex-valued output field distribution. Intensity distributions at different depths from the meta-optic output were then magnified with an objective and tube lens and recorded with a camera. Amplitude control over the incident optical field can be directly verified by imaging the output plane of the meta-optic. If the output field phase distribution is accurate, propagation of the output field will form the desired intensity profile at each plane in space beyond the meta-optic. However, if the phase distribution is inaccurate, the desired intensity will not be formed at a distance beyond the meta-optic. Therefore, phase control was verified by comparing the measured intensity distribution at a plane beyond the meta-optic output.

To demonstrate the accuracy of the compound meta-optic in providing independent phase and amplitude control with high efficiency, two different functions are presented. First, we show a combined 
beam-forming and splitting function where a uniform illumination is reshaped to form multiple output beams with specified amplitude profiles and propagation directions. Second, we design meta-optics to reshape a uniform illumination to form computer-generated, three-dimensional holograms.

\section{Meta-optics for combined beam-forming and splitting}

Using the meta-optic design process ${ }^{24,25}$, we designed, fabricated, and measured two meta-optic devices performing optical beam-forming and splitting at a wavelength of $\lambda_{0}=1.3 \mu \mathrm{m}$. In both examples, a circular uniform illumination with diameter of $200 \mu m$ is reshaped to form multiple output Gaussian beams. Each metasurface is $200 \mu \mathrm{m}$ in diameter and separated by $325 \mu \mathrm{m}$ of PDMS. The first meta-optic forms the interference pattern between two Gaussian beams of different beamwidths, propagation directions, and relative intensities. The desired output field profile is calculated as the superposition of the two Gaussian beams as

$$
E_{\text {out }}=-e^{-\left(r / 45.5 \lambda_{0}\right)^{2}} e^{i k_{0} x \sin \left(-2^{\circ}\right)}+\sqrt{0.5} e^{-\left(r / 32.5 \lambda_{0}\right)^{2}} e^{i k_{0} x \sin \left(2^{\circ}\right)}
$$

which forms a fringed interference pattern. Since the exact complex-valued field distribution is formed, only the desired Gaussian beams are produced, and no undesired diffraction orders are present. Each metasurface of the device was simulated using the open-source finite-difference time-domain (FDTD) solver $\mathrm{MEEP}^{39}$, and their responses combined to determine the overall meta-optic output field distribution (additional information about the meta-optic designs can be found in Supplementary Note 3). Figure 3(a) compares the intensity profiles formed by the meta-optic at the output plane and at a far-field distance for simulation and measurement. In each case, we see that the measurements closely agree with the simulated intensity distributions and form the desired Gaussian beams with little diffractive noise. Furthermore, the transformation in amplitude and phase was performed with an efficiency of $81 \%$ in simulation and $72 \%$ in measurement. The efficiency is defined as the fraction of intensity in the uniform illumination contained within the beam cross-sections (with Fresnel reflection correction). 

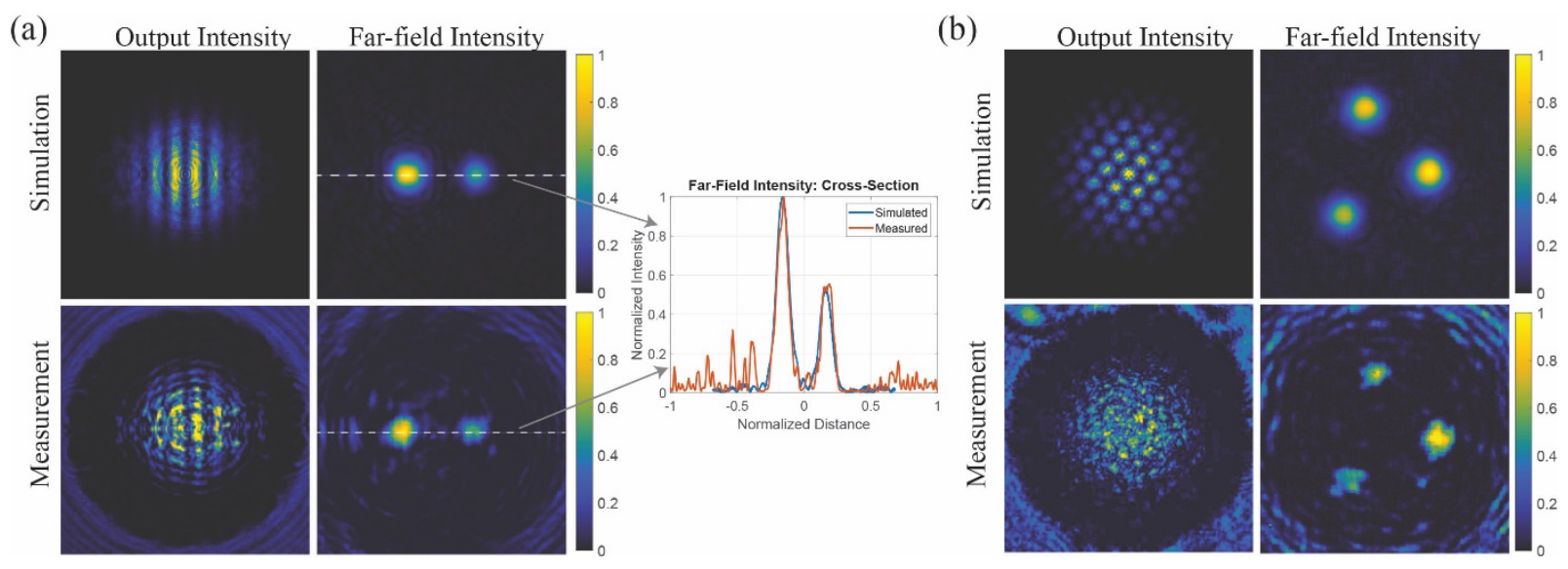

Figure 3: Measurement results of meta-optics implementing a beam-forming and splitting function, where a uniform circular illumination is manipulated to form multiple Gaussian beams at the output. In each case, measurements closely match the simulation results. Note that intensities have been normalized to respective maximums. (a) Two Gaussian beams are formed by the meta-optic. The output intensity is the characteristic fringing pattern of two interfering beams, verifying that the amplitude distribution of the optical field has been reshaped. The two beams appear separate at a far-field distance, verifying that the phase distribution at the output is also correct. A cross-section through the beams is taken to show that the Gaussian profile of each beam is accurately formed. (b) Three Gaussian beams are formed by a meta-optic. The output intensity is the interference pattern between the three beams and verifies that the amplitude profile has been reshaped. Three separate beams are observed at a far-field distance, matching simulation results. (Perceptually uniform color bar ${ }^{40}$ used for these and all following plots).

A second beam-former/splitter example was fabricated to produce three output Gaussian beams. The propagation directions are chosen such that the output field has variation in both planar dimensions (see Supplementary Note 3 for design specifics). Figure 3(b) compares the intensity profiles formed by the meta-optic at the output plane and a far-field distance in simulation to measurements. Here, the desired Gaussian beams are formed at the correct relative intensities, propagation directions, and beamwidths, verifying the accuracy of amplitude and phase control performed by the meta-optic. The device efficiency is $79 \%$ in simulation and $65 \%$ in measurement. One reason for the slight reduction in experimental efficiency of the beam-splitter devices is an experimentally observed transmission dip for 
pillars with diameters in the $310 \mathrm{~nm}-330 \mathrm{~nm}$ range. This feature is discussed in more detail in Supplementary Note 1 .

\section{Meta-optics for three-dimensional holograms}

Another exciting application of high-efficiency amplitude and phase control is three-dimensional holography. While phase-only metasurfaces can produce holograms, phase and amplitude control allows for enhanced image quality, especially for three-dimensional holograms ${ }^{13,16}$. Such complex-valued holograms have been generated using lossy methods, most commonly reflection ${ }^{22}$ or polarization loss ${ }^{14-17}$. Here, we utilize the meta-optic design approach to demonstrate high-efficiency, three-dimensional holograms. Multiple computer-generated hologram approaches can be used to display a three-dimensional scene $^{41}$, but we demonstrate two methods: a simple point-source hologram and a hologram composed of solid flat image components ${ }^{42-44}$ more applicable to generating a realistic hologram scene.

Point-source holograms approximate the surface of a three-dimensional object with a collection of point sources and have the advantage of wide viewing angles due to the diverging spherical wave from each point source. The field distribution at the meta-optic output that forms the hologram is calculated by summing the complex conjugated radiated fields from each point source. Phase-only metasurfaces can implement such holograms by directly applying the phase of this field distribution to the incident field to form images. However, image quality is notably improved if the amplitude pattern is also formed.

Here, a compound meta-optic is designed to form the complex-valued interference field of point sources tracing the outline of the University of Michigan and Vanderbilt University logos. Each logo is tilted about its center by 15 degrees to provide depth to the image, as shown by the diagram in Figure 4(a). The metasurfaces, designed to operate at $\lambda_{0}=1.1 \mu \mathrm{m}$, were square arrays $200 \mu \mathrm{m}$ in extent and separated by a distance of $275 \mu m$ (see Supplementary Note 3 for additional design details and different unit cell dimensions). Figure 4(b) compares the simulated hologram to the measured hologram at different 
depths, where the in-focus portion of each image is marked with a red arrow. We see that the measured intensity images closely match the desired intensity images with very little speckle noise.

(a)
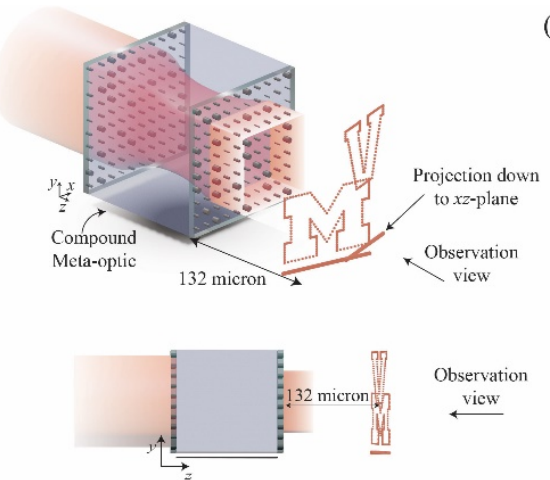

(b)
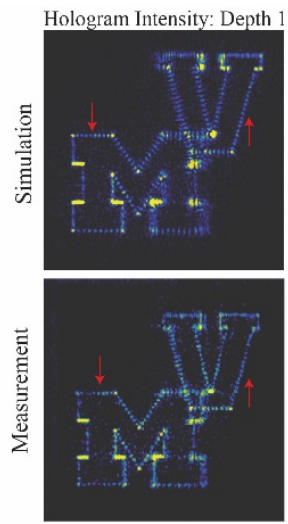
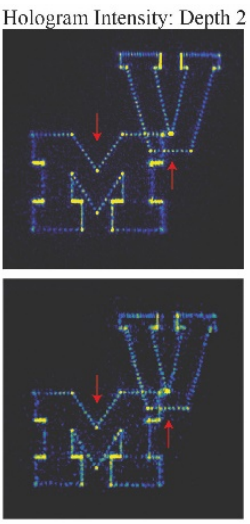
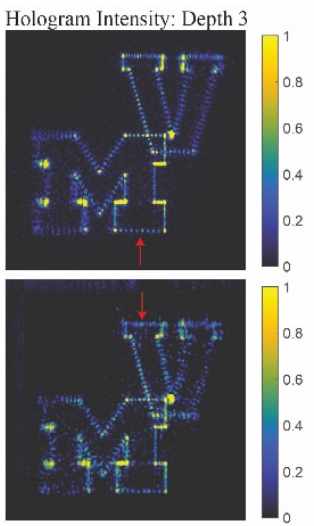

(c)

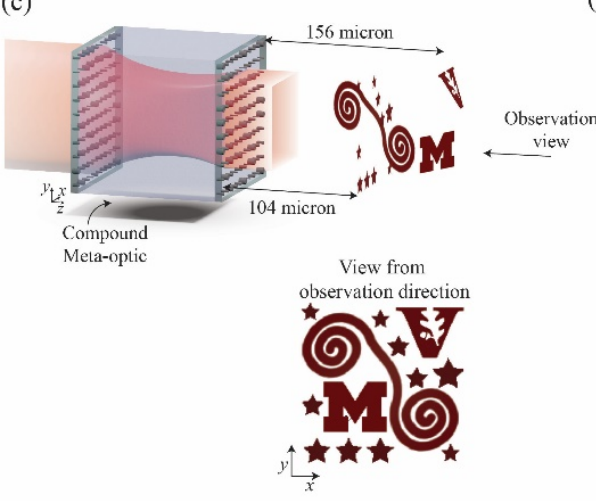

(d)

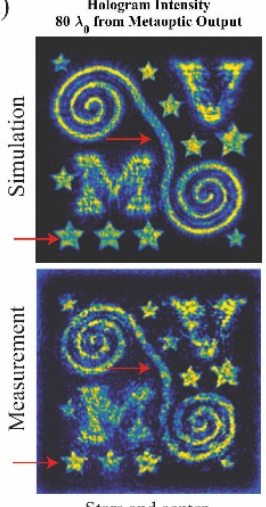

Stars and center

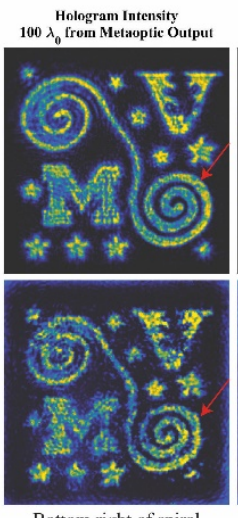

Bottom right of spiral

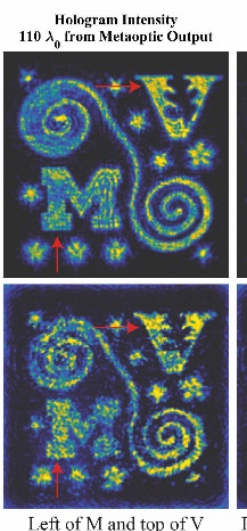

Left of $\mathrm{M}$ and top of $\mathrm{V}$ in focus

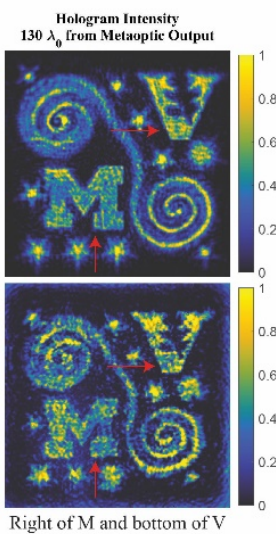

in focus

Figure 4: Comparison of simulation and measurement results of compound meta-optics implementing two 3D holograms. In each case, the measurement results closely match the expected simulation results. Note that intensities are normalized to respective maximums. (a) A point source hologram formed by a compound meta-optic at $\lambda_{0}=1.1 \mu m$. The University of Michigan and Vanderbilt University logos are tilted in space by 15 degrees, providing depth to the hologram. (b) The hologram intensity was recorded at different distances from the output plane of the compound meta-optic, showing that different portions of the hologram are in-focus at different depths (as denoted by the red arrows). (c) A 3D hologram using solid image components is formed by a compound meta-optic at $\lambda_{0}=1.3 \mu \mathrm{m}$. The large spiral, University of Michigan logo, and Vanderbilt University logo are tilted in space to provide depth to the scene, while the stars are formed in the same plane, at the halfway point of the spiral. (d) The hologram intensity was recorded at different distances from the meta-optic output, showing the different image components coming into and out of focus. 
While point-source holograms have wide viewing angles, they are unable to visually re-create the appearance of a realistic scene due to the point-based sampling of the surface. Next, we use computergenerated hologram techniques to generate a three-dimensional hologram consisting of solid image

components tilted in space ${ }^{42-44}$. These techniques can be used to produce a faceted representation of an object, leading to the ability of forming $3 \mathrm{D}$ holograms of life-like scenes ${ }^{25,42-44}$.

For this example, we designed a compound meta-optic where each metasurface is a $200 \mu \mathrm{m}$ square array of nanopillars and separated by $325 \mu \mathrm{m}$ of PDMS. The operating wavelength is $\lambda_{0}=1.3 \mu \mathrm{m}$. The output field of the compound meta-optic was engineered in amplitude and phase to form the scene shown in Figure 4(c). The large spiral, University of Michigan (M), and Vanderbilt University (V) logos are all tilted in space, but in different directions (see Supplementary Note 3 for additional design details). As a result, different cross-sections of the image come into focus when imaging different depths from the meta-optic output plane. Figure 4(d) shows intensity images measured at different depths in the 3D hologram, which closely match the simulated images. Specifically, the first image shows the middle of the spiral and stars in focus, while the remaining images show different cross-sections of the logos. The full three-dimensional nature of the hologram can be seen by scanning the focal plane through the volume of the hologram (see Supplementary Movie 1). The compound meta-optic performs the desired complexvalued field manipulation with a simulated efficiency of $82 \%$ and measured efficiency of $75 \%$ for this example, significantly exceeding the efficiency of loss-based approaches ${ }^{14-16,22}$. The efficiency considered here is the percentage of the input intensity present in the transmitted field distribution at the output of the meta-optic.

\section{Discussion}

The measured intensity images from each of the meta-optic devices demonstrate the accuracy of complex-valued optical field control with high efficiency. Specifically, the intensity measured at the output plane of the meta-optic demonstrates amplitude control since the uniform illumination is reshaped. 
The intensity measured at a plane beyond the aperture (after the optical field propagates away from the meta-optic output) demonstrates phase control since the desired intensity will only be formed if the output phase profile is accurate. An inaccurate phase profile would alter the propagation characteristics of the optical field so that the measured intensity pattern would not match the desired result. This is verified in Figure 3, where combined beam-former and splitters are demonstrated to form intensity distributions which closely match the expected distributions. Even though a small number of Gaussian beams were formed with these devices, meta-optics can be designed to produce a larger number of beams with different amplitude profiles ${ }^{25}$. The measured efficiency of the beam-former/splitter designs of $78 \%$ and $65 \%$ is higher than that of methods which would require loss.

Similarly, the measured three-dimensional holograms shown in Figure 4 very closely match the desired intensity images, demonstrating that the meta-optics can produce life-like holograms. The images produced in Figure 4(d) with phase and amplitude control avoid the image speckle common to phase-only implementations produced with phase-retrieval algorithms ${ }^{16}$. Furthermore, scanning the imaging plane through the hologram shows the image components are accurately reproduced at the desired spatial locations (see Supplementary Movie 1). This approach also maintains the image contrast compared to the desired phase profile simply applied to the uniform illumination (see Supplementary Note 4 for comparison). While the fabricated meta-optics demonstrated relatively simple holograms, meta-optics can be designed to form 3D holograms of life-like scenes ${ }^{25}$.

High-efficiency amplitude and phase control over optical fields enables compound meta-optics to explicitly perform a variety of applications without inherent losses in efficiency. In contrast, loss-based amplitude control significantly reduces the device efficiency. For example, a measured efficiency of $6.4 \%$ was demonstrated in forming a complex-valued hologram using polarization conversion at $\mathrm{THz}$ frequencies ${ }^{17}$ (however, intrinsic unit cell losses also contributed, since the measured efficiency for a similar phase-only version was $19.1 \%$ ). Even when unit cells are optimized to obtain a maximum 
polarization conversion efficiency of $100 \%{ }^{16}$, the overall efficiency of a device is less than unity and highly case-dependent due to variation of transmission amplitude over the metasurface. Therefore, there are two main advantages of the compound meta-optic approach in terms of efficiency. First, using polarization loss for amplitude control requires a specific input polarization, which decreases the overall efficiency if an unpolarized source is used. Instead, the method shown here is polarization independent and avoids this issue. Second, the efficiency of loss-based approaches is highly case-dependent, but compound meta-optics can achieve near-unity efficiency (in the ideal case) regardless of the required amplitude control. As a result, the measured $75 \%$ efficiency of the compound meta-optic producing the solid-image 3D hologram is significantly higher than lossy methods ${ }^{14-17}$ and is approximately independent of the desired amplitude control.

The reduced measured efficiency relative to simulation is attributed to fabrication errors, in particular an experimental transmission dip for pillars with diameters in the $310 \mathrm{~nm}-330 \mathrm{~nm}$ range (in the case of the beam-splitting devices), and misalignment of the two metasurfaces. Fabrication errors and misalignment can also lead to phase errors in the output field distribution, causing slight degradation in image quality observed between simulation and experiment. Additionally, since a locally periodic approximation was used to design the metasurfaces, differences between the local periodic performance of each unit cell and inhomogeneous metasurface performance contribute to reduced efficiency and image quality reduction. Optimizing the inhomogeneous metasurface structure ${ }^{45,46}$ could lead to improvements in efficiency and transmission phase accuracy. That said, the demonstrated compound meta-optics already exhibits amplitude and phase control with high efficiency and accuracy.

In conclusion, we have demonstrated compound meta-optics consisting of paired, lossless metasurfaces that independently manipulate the amplitude and phase distributions of an optical field. Each metasurface was implemented as a high-transmission array of amorphous silicon nanopillars and aligned, operating at near-infrared wavelengths. The distance between the metasurfaces allows the optical 
wave to be reshaped, leading to high-efficiency devices by avoiding loss-based amplitude-control mechanisms. High-efficiency field control expands the application space of meta-optics, while maintaining a compact form-factor. As examples, we have experimentally shown that compound metaoptics can implement optical functions such as combined beam-forming and splitting, as well as form three-dimensional holograms with high image quality. In each case, the measured efficiency of the fabricated meta-optic devices ranged between 65\%-75\%. By explicitly forming the desired complexvalued field profiles, no energy is lost to diffraction and high-quality holograms are obtained. The compound meta-optic approach can be extended to visible optical wavelengths by a change in materials.

For example, titanium dioxide can be used in place of silicon to fabricate the nanopillars ${ }^{5,47}$. Additionally, polarization control can be achieved through an anisotropic nanopillar cross-section. Compound metaoptics could lead to improved performance in three-dimensional holography, compact holographic displays, custom optical elements, and other applications requiring detailed control over the phase, amplitude, and polarization distributions of an optical field.

\section{Methods}

\section{Simulation}

An open-source finite difference time domain (FDTD) solver $\mathrm{MEEP}^{39}$ was used to simulate the transmission performance of the silicon nanopillar unit cells and each metasurface composing the metaoptics. The unit cells were individually simulated with periodic boundary conditions, while the metasurface simulation used perfectly matched layer (PML) boundaries $2 \mu m$ from the metasurface edge. The source field distribution was placed $1.5 \mu \mathrm{m}$ in front of the metasurface or unit cell, and a monitor placed $1.5 \mu \mathrm{m}$ after to record the transmission field amplitude and phase. To simulate the compound metaoptic, the transmitted field from the simulation of metasurface 1 was numerically propagated across the separation distance and used as the source for the simulation of metasurface $2^{25}$. The transmitted field 
from the FDTD simulation of metasurface 2 is considered the output field of the meta-optic and compared to the measurement results.

\section{Fabrication}

The metasurface patterns were defined on an 850nm-thick amorphous Si wafer, which was grown on a fused silica substrate using low-pressure chemical vapor deposition (LPCVD). A 200nm-thick PMMA A4 was spin coated at $4500 \mathrm{rpm}$, followed by deposition of $10 \mathrm{~nm}$-thick chromium as the conduction layer using thermal evaporation. The metasurface structures were defined using electron beam lithography (EBL), followed by the deposition of $35 \mathrm{~nm}$-thick aluminum oxide as the dry etch mask using e-beam evaporation. The Si nanoposts were then formed by reactive ion etching (RIE) using a mixture of $\mathrm{SF}_{6}$ and $\mathrm{C}_{4} \mathrm{~F}_{8}$.

To completely encapsulate the Si nanoposts, the first layer of PMDS (10:1 mixing ratio of Sylgard 184 base and curing agent) was diluted in toluene in a 2:3 weight ratio, which was spin coated at $2500 \mathrm{rpm}$ and cured at $80 \mathrm{C}^{\circ}$ for more than half an hour. The second layer of PMDS without dilution was subsequently spin coated and cured at $80 \mathrm{C}^{\circ}$ for more than 2 hours. The same procedure was used for both metasurface layers.

\section{Measurement}

A customized alignment system is shown in Figure $2 \mathrm{~b}$. The bottom metasurface layer was mounted on a rotation stage held by a vacuum pump, and the top layer was attached to a glass slide suspended by an XYZ translation stage. A drop of uncured PMDS was applied in between as an indexmatched layer. The samples were then illuminated from the bottom by a collimated supercontinuum laser that was passed through a monochromator set at the designed working wavelength. The far-field images after the bilayer metasurface were recorded by a NIR camera through an imaging system consisting of a 20X objective and a tube lens. The alignment holograms were then aligned by tuning the XYZ translation and rotation stage. After spatial alignment was achieved, the XY translation was further adjusted until the 
desirable far-field images were formed. The efficiency was calculated based on the images captured by the NIR camera with the background noise correction processed by subtraction of a blank image, which contained the dark current signals. The reference intensity was calculated from images of the substrates without any meta-optics. With the same integration time, the intensity distribution can be revealed by the photon count from the camera. The efficiency of the beam-former/splitter devices was calculated as the intensity within an area encompassing each beam in the far-field, divided by the intensity incident on the device. For the three-dimensional hologram example, the efficiency is the intensity contained in the transmitted field divided by the intensity incident on the device.

\section{References}

1. Yu, N. \& Capasso, F. Flat optics with designer metasurfaces. Nat. Mater. 13, (2014).

2. Staude, I. \& Schilling, J. Metamaterial-inspired silicon nanophotonics. Nat. Photonics 11, 274 284 (2017).

3. He, Q., Sun, S., Xiao, S. \& Zhou, L. High-Efficiency Metasurfaces: Principles, Realizations, and Applications. Advanced Optical Materials vol. 6 (2018).

4. Kamali, S. M., Arbabi, E., Arbabi, A. \& Faraon, A. A review of dielectric optical metasurfaces for wavefront control. Nanophotonics 7, 1041-1068 (2018).

5. Khorasaninejad, M. et al. Metalenses at visible wavelengths: Diffraction-limited focusing and subwavelength resolution imaging. Science (80-. ). 352, 1190-1194 (2016).

6. Zhou, Y. et al. Multilayer Noninteracting Dielectric Metasurfaces for Multiwavelength Metaoptics. Nano Lett. 18, 7529-7537 (2018).

7. Zhang, D. et al. Nanoscale beam splitters based on gradient metasurfaces. Opt. Lett. 43, 267 (2018).

8. Zhang, X. et al. Metasurface-Based Ultrathin Beam Splitter with Variable Split Angle and Power Distribution. ACS Photonics 5, 2997-3002 (2018).

9. Li, J. et al. Efficient Polarization Beam Splitter Based on All-Dielectric Metasurface in Visible Region. Nanoscale Res. Lett. 14, 34 (2019).

10. Lin, Y., Wang, M., Sui, Z., Zeng, Z. \& Jiang, C. Highly efficient beam splitter based on alldielectric metasurfaces. Jpn. J. Appl. Phys. 58, 060918 (2019).

11. Liu, L. et al. Broadband metasurfaces with simultaneous control of phase and amplitude. $A d v$. Mater. 26, 5031-5036 (2014).

12. Song, X. et al. Selective Diffraction with Complex Amplitude Modulation by Dielectric Metasurfaces. Adv. Opt. Mater. 6, 1701181 (2018).

13. Huang, L., Zhang, S. \& Zentgraf, T. Metasurface holography: From fundamentals to applications. Nanophotonics 7, 1169-1190 (2018).

14. Huang, L. et al. Three-dimensional optical holography using a plasmonic metasurface. Nat. Commun. 4, 1-8 (2013). 
15. Lee, G. Y. et al. Complete amplitude and phase control of light using broadband holographic metasurfaces. Nanoscale 10, 4237-4245 (2018).

16. Overvig, A. C. et al. Dielectric metasurfaces for complete and independent control of the optical amplitude and phase. Light Sci. Appl. 8, 2047-7538 (2019).

17. Wang, Q. et al. Broadband metasurface holograms: Toward complete phase and amplitude engineering. Sci. Rep. 6, 1-10 (2016).

18. Lee, G. Y. et al. Metasurface eyepiece for augmented reality. Nat. Commun. 9, 1-10 (2018).

19. Joo, W.-J. et al. Metasurface-driven OLED displays beyond 10,000 pixels per inch. Science (80-. ). 370, 459-463 (2020).

20. Zhou, Y., Zheng, H., Kravchenko, I. I. \& Valentine, J. Flat optics for image differentiation. Nat. Photonics 14, 316-323 (2020).

21. Faraji-Dana, M. et al. Compact folded metasurface spectrometer. Nat. Commun. 9, (2018).

22. Zhou, Y. et al. Multifunctional metaoptics based on bilayer metasurfaces. Light Sci. Appl. 8, 2047-7538 (2019).

23. Kwon, D. H., Ptitcyn, G., Díaz-Rubio, A. \& Tretyakov, S. A. Transmission Magnitude and Phase Control for Polarization-Preserving Reflectionless Metasurfaces. Phys. Rev. Appl. 9, 034005 (2018).

24. Raeker, B. O. \& Grbic, A. Compound Metaoptics for Amplitude and Phase Control of Wave Fronts. Phys. Rev. Lett. 122, (2019).

25. Raeker, B. O. \& Grbic, A. Lossless, Complex-Valued Optical Field Control with Compound Metaoptics. arXiv (preprint at https://arxiv.org/abs/2011.07185) (2020).

26. Backer, A. S. Computational inverse design for cascaded systems of metasurface optics. Opt. Express 27, 30308 (2019).

27. Ataloglou, V. G., Dorrah, A. H. \& Eleftheriades, G. V. Design of Compact Huygens' Metasurface Pairs With Multiple Reflections for Arbitrary Wave Transformations. IEEE Trans. Antennas Propag. 68, (2020).

28. Brown, T. \& Mojabi, P. Cascaded Metasurface Design Using Electromagnetic Inversion with Gradient-Based Optimization. TechRxiv (preprint) (2020) doi:10.36227/TECHRXIV.12743663.V1.

29. Arbabi, A. et al. Miniature optical planar camera based on a wide-angle metasurface doublet corrected for monochromatic aberrations. Nat. Commun. 7, 443-803 (2016).

30. Arbabi, A., Arbabi, E., Horie, Y., Kamali, S. M. \& Faraon, A. Planar metasurface retroreflector. Nat. Photonics 11, 415-420 (2017).

31. Huang, X., Shrestha, S., Overvig, A. \& Yu, N. Three-Color Phase-Amplitude Holography with a Metasurface Doublet - IEEE Conference Publication. in 2020 Conference on Lasers and ElectroOptics (CLEO) 1-2 (2020).

32. Lin, X. et al. All-optical machine learning using diffractive deep neural networks. Science (80-. ). 361, 1004-1008 (2018).

33. Qian, C. et al. Performing optical logic operations by a diffractive neural network. Light Sci. Appl. 9, 2047-7538 (2020).

34. Gerchberg, R. W. \& Saxton, W. O. A Practical Algorithm for the Determination of Phase from Image and Diffraction Plane Pictures. Opt. 35, 237-246 (1972).

35. Arbabi, A., Horie, Y., Bagheri, M. \& Faraon, A. Dielectric metasurfaces for complete control of 
phase and polarization with subwavelength spatial resolution and high transmission. Nat. Nanotechnol. 10, (2015).

36. Balthasar Mueller, J. P., Rubin, N. A., Devlin, R. C., Groever, B. \& Capasso, F. Metasurface Polarization Optics: Independent Phase Control of Arbitrary Orthogonal States of Polarization. Phys. Rev. Lett. 118, 113901 (2017).

37. Pfeiffer, C. \& Grbic, A. Cascaded metasurfaces for complete phase and polarization control. Appl. Phys. Lett. 102, (2013).

38. Pestourie, R. et al. Inverse design of large-area metasurfaces. Opt. Express 26, (2018).

39. Oskooi, A. F. et al. Meep: A flexible free-software package for electromagnetic simulations by the FDTD method. Comput. Phys. Commun. 181, 687-702 (2010).

40. Kovesi, P. Good Colour Maps: How to Design Them. (2015).

41. Park, J.-H. Recent progress in computer-generated holography for three-dimensional scenes. J. Inf. Disp. 18, 1-12 (2017).

42. Matsushima, K. \& Nakahara, S. Extremely high-definition full-parallax computer-generated hologram created by the polygon-based method. Appl. Opt. 48, H54-H63 (2009).

43. Matsushima, K. Computer-generated holograms for three-dimensional surface objects with shade and texture. Appl. Opt. 44, 4607-4614 (2005).

44. Matsushima, K., Nishi, H. \& Nakahara, S. Simple wave-field rendering for photorealistic reconstruction in polygon-based high-definition computer holography. J. Electron. Imaging 21, 1 (2012).

45. Phan, T. et al. High-efficiency, large-area, topology-optimized metasurfaces. Light Sci. Appl. 8 , (2019).

46. Mansouree, M. et al. Multifunctional 25D metastructures enabled by adjoint optimization. Optica 7, 77 (2020).

47. Devlin, R. C., Khorasaninejad, M., Chen, W. T., Oh, J. \& Capasso, F. Broadband high-efficiency dielectric metasurfaces for the visible spectrum. Proc. Natl. Acad. Sci. U. S. A. 113, 10473-10478 (2016).

\section{Acknowledgements}

B.O.R. and A.G. acknowledge financial support by the National Science Foundation Graduate Research Fellowship Program. B.O.R, H.Z., Y.Z., J.V., and A.G. acknowledge financial support by the Office of Naval Research under Grant No. N00014-18-1-2536. Additionally, this research was supported in part through computational resources and services provided by Advanced Research Computing at the University of Michigan, Ann Arbor. A portion of this research was conducted at the Center for Nanophase Materials Sciences, which is a DOE Office of Science User Facility. Finally, B.O.R. and A.G. are grateful to Cody Scarborough for assistance in creating graphics seen here. 


\section{Additional information}

\section{Competing Interests}

The authors declare no competing interests. 


\section{Figures}
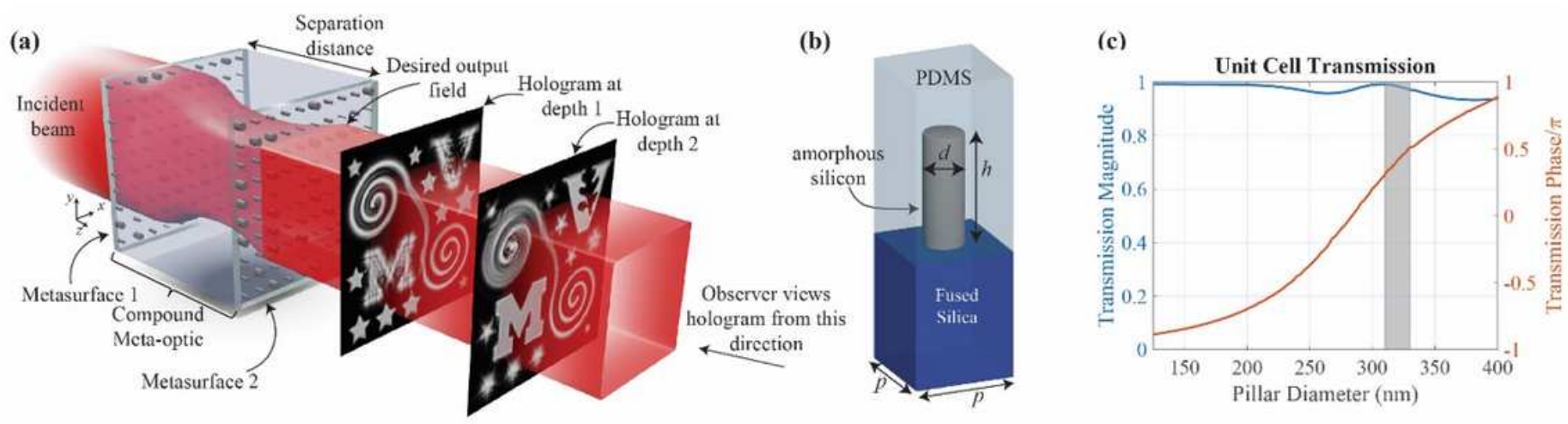

\section{Figure 1}

Diagram of the optical compound meta-optic implementing independent phase and amplitude control over an incident optical field. (a) Illustration of a compound meta-optic converting a uniform illumination into a three-dimensional, complex-valued hologram. Depth of the three-dimensional hologram is visualized when the output field is imaged at different depths by an observer facing the device. (b) A schematic of the metasurface unit cell. Each cell has a period $p=570 \mathrm{~nm}$, and contains an amorphous silicon nanopillar with a height $h=850 \mathrm{~nm}$. The nanopillar diameter is varied across the metasurface to implement the desired transmission phase profile. (c) The transmission characteristics of the unit cell under normally incident plane wave illumination and periodic boundary conditions. A phase coverage of $78 \%$ with transmission above 0.93 is achieved by varying the pillar diameter. Diameters of $310 \mathrm{~nm}-330 \mathrm{~nm}$ are not used in the three-dimensional hologram designs due to observed low transmission in fabrication, which impact the image quality.

(a)

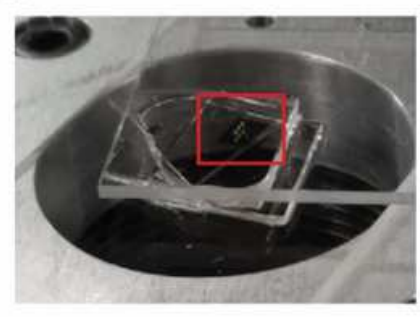

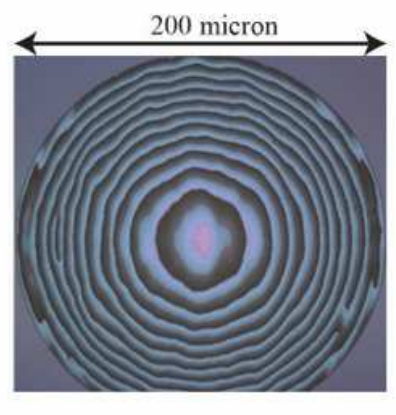

(b)

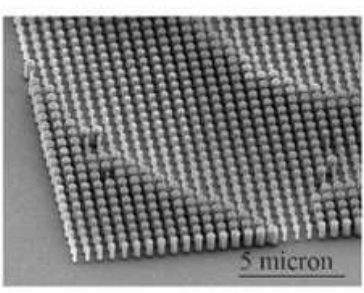

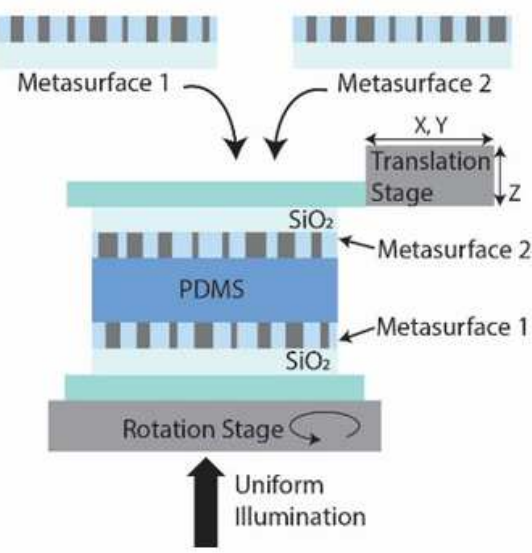

\section{Figure 2}

Images of a fabricated meta-optic and schematic of meta-optic assembly. (a) Left: An optical image of the fabricated meta-optic device, where the two metasurface layers are being aligned. Middle: Optical 
image of a fabricated metasurface, showing the variation in pillar diameter as a change in color. Right: A scanning electron microscope image of a portion of one metasurface, showing the array of silicon nanopillars. (b) Schematic of the meta-optic assembly, alignment, and characterization process. Each metasurface is fabricated individually and then aligned to form the meta-optic device.
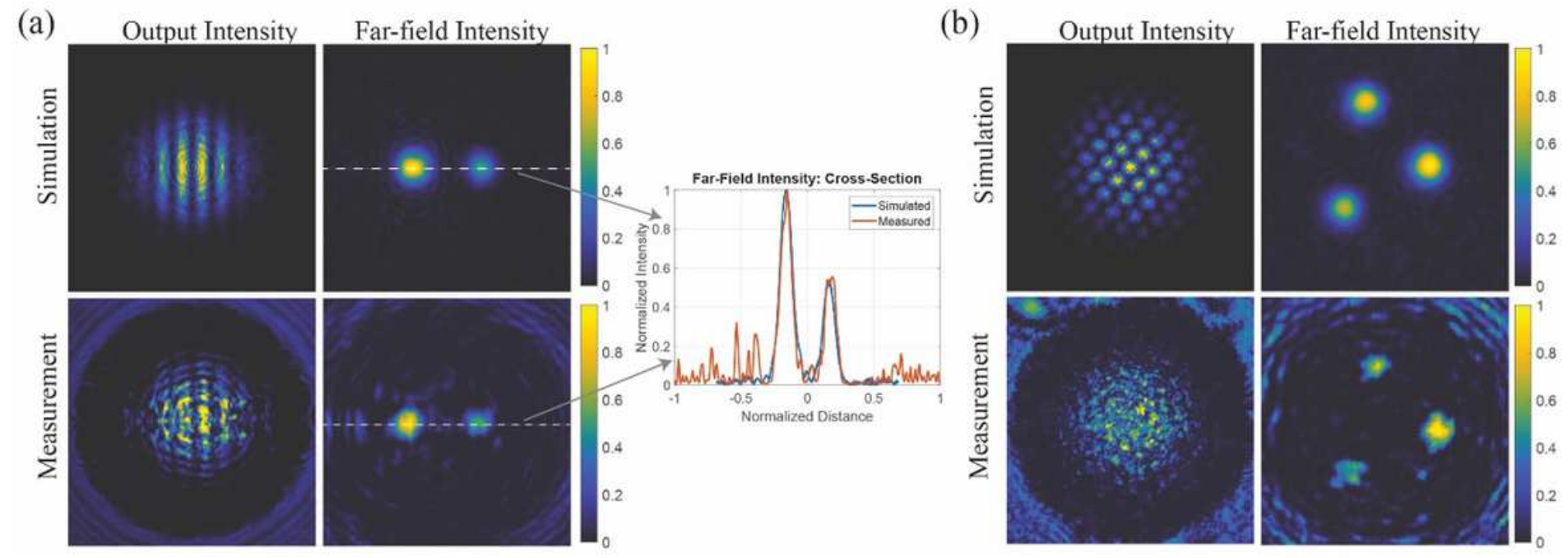

\section{Figure 3}

Measurement results of meta-optics implementing a beam-forming and splitting function, where a uniform circular illumination is manipulated to form multiple Gaussian beams at the output. In each case, measurements closely match the simulation results. Note that intensities have been normalized to respective maximums. (a) Two Gaussian beams are formed by the meta-optic. The output intensity is the characteristic fringing pattern of two interfering beams, verifying that the amplitude distribution of the optical field has been reshaped. The two beams appear separate at a far-field distance, verifying that the phase distribution at the output is also correct. A cross-section through the beams is taken to show that the Gaussian profile of each beam is accurately formed. (b) Three Gaussian beams are formed by a meta-optic. The output intensity is the interference pattern between the three beams and verifies that the amplitude profile has been reshaped. Three separate beams are observed at a far-field distance, matching simulation results. (Perceptually uniform color bar40 used for these and all following plots). 
(a)
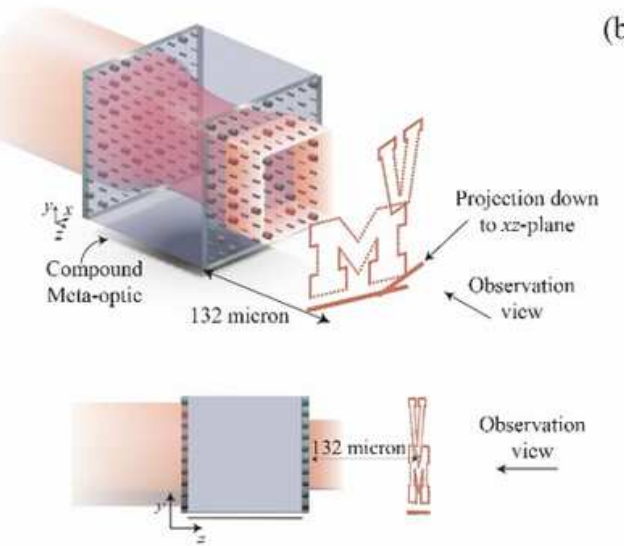

(b)

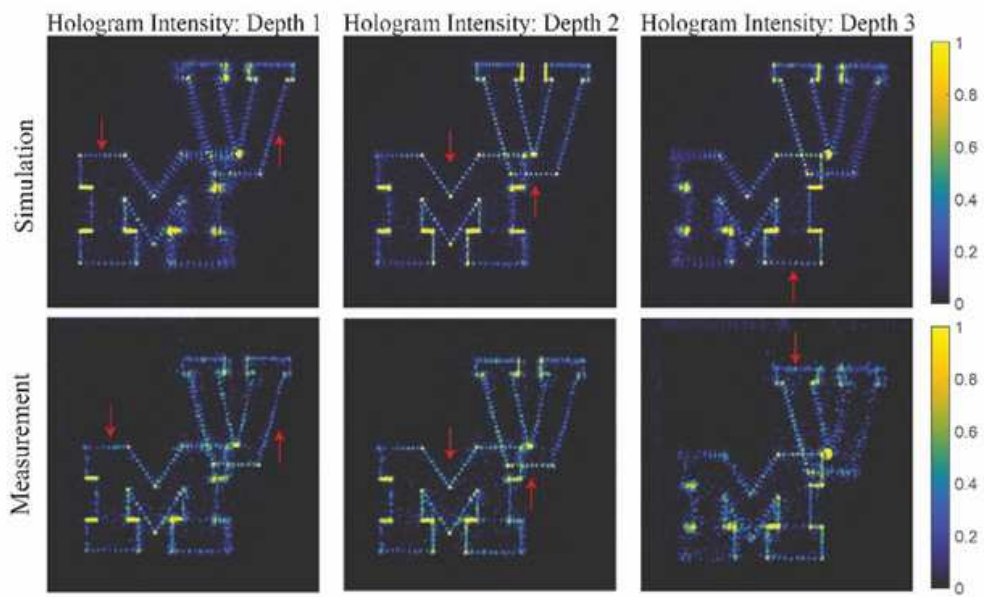

(c)

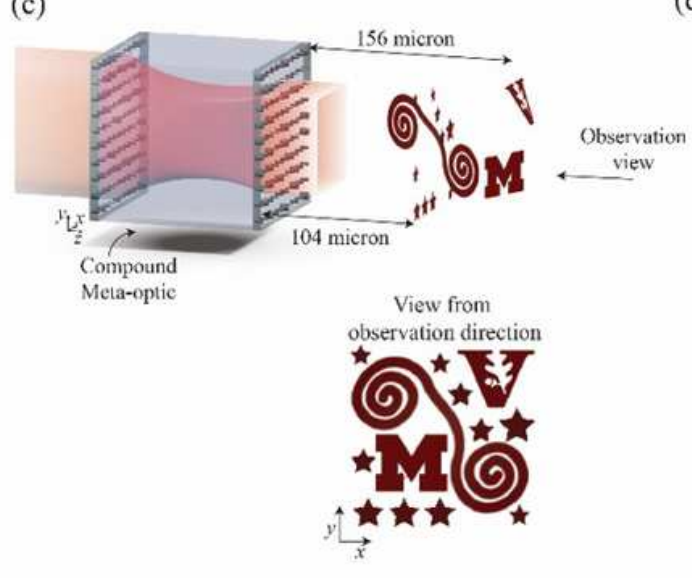

(d)
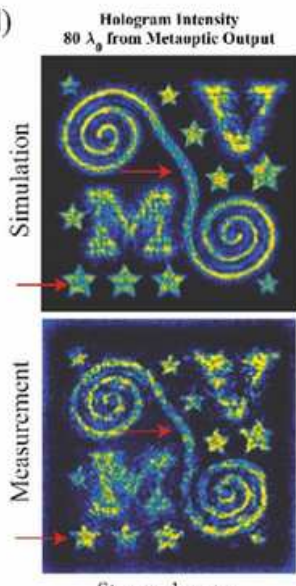

Stars and center of spiral in focus

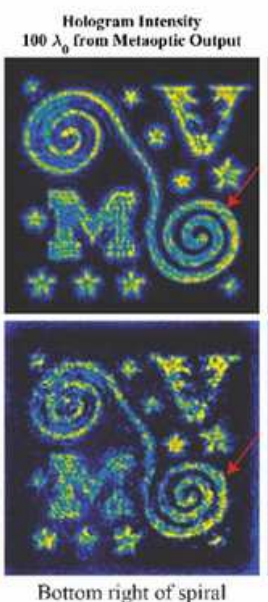

in focus

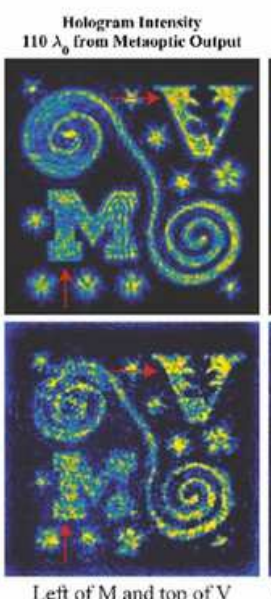

in focus

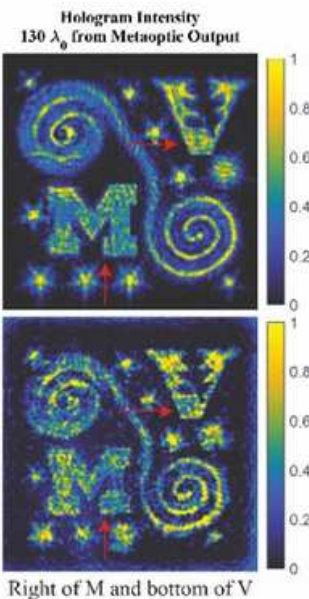

in focus

\section{Figure 4}

Comparison of simulation and measurement results of compound meta-optics implementing two 3D holograms. In each case, the measurement results closely match the expected simulation results. Note that intensities are normalized to respective maximums. (a) A point source hologram formed by a compound meta-optic at $\lambda \_0=1.1 \mu \mathrm{m}$. The University of Michigan and Vanderbilt University logos are tilted in space by 15 degrees, providing depth to the hologram. (b) The hologram intensity was recorded at different distances from the output plane of the compound meta-optic, showing that different portions of the hologram are in-focus at different depths (as denoted by the red arrows). (c) A 3D hologram using solid image components is formed by a compound meta-optic at $\lambda \_0=1.3 \mu \mathrm{m}$. The large spiral, University of Michigan logo, and Vanderbilt University logo are tilted in space to provide depth to the scene, while the stars are formed in the same plane, at the halfway point of the spiral. (d) The hologram intensity was recorded at different distances from the meta-optic output, showing the different image components coming into and out of focus.

\section{Supplementary Files}


This is a list of supplementary files associated with this preprint. Click to download.

- NIRMetaopticPaperSupplemental.docx

- SupplementalMovie1.mp4 